\title{
A Novel Blind Source Separation Algorithm and Performance Analysis of Weak Signal against Strong Interference in Passive Radar Systems
}

\author{
Chengjie Li, Lidong Zhu, Anhong Xie, and Zhongqiang Luo \\ National Key Laboratory of Science and Technology on Communications, University of Electronic Science and \\ Technology of China, No. 2006, Xiyuan Avenue, West Hi-Tech Zone, Chengdu 610000, China
}

Correspondence should be addressed to Chengjie Li; junhongabc@126.com

Received 30 November 2015; Accepted 3 March 2016

Academic Editor: Sotirios K. Goudos

Copyright (C) 2016 Chengiie Li et al. This is an open access article distributed under the Creative Commons Attribution License, which permits unrestricted use, distribution, and reproduction in any medium, provided the original work is properly cited.

\begin{abstract}
In Passive Radar System, obtaining the mixed weak object signal against the super power signal (jamming) is still a challenging task. In this paper, a novel framework based on Passive Radar System is designed for weak object signal separation. Firstly, we propose an Interference Cancellation algorithm (IC-algorithm) to extract the mixed weak object signals from the strong jamming. Then, an improved FastICA algorithm with $K$-means cluster is designed to separate each weak signal from the mixed weak object signals. At last, we discuss the performance of the proposed method and verify the novel method based on several simulations. The experimental results demonstrate the effectiveness of the proposed method.
\end{abstract}

\section{Introduction}

Passive Radar System is an object signal detection system that does not generate a radiofrequency signal itself but only receives the detected target signal [1]. An example of the Passive Radar System is shown in Figure 1. We can see it is a kind of signal detection and analysis system that obtains object signal information from the radiation source.

The signals in Passive Radar System are composed of two parts: interference signal and mixed object signals. Since the interference signal is stronger (strong interference signal) compared with the object signals (weak object signal), it is very difficult to obtain the weak object signals against the strong interference signal. Meanwhile, the object signals are mixed with several signals. Separating each one from the mixed object signals is another challenging task.

Several existing algorithms are partially related to the object signal detection, such as the Relax algorithm by Jian et al. [2, 3], CLEAN technology by Gough [4], FFT signal separation method by Ziskind and Wax [5], JJM algorithm [6], and FastICA algorithm by Hyvärinen et al. [7-9]. Although these algorithms [2-6] are partially related to the weak signal separation, their performances on passive communication system are still not sufficient for practical applications. Hence, it is still necessary to develop more efficient object signal detection algorithm for the Passive Radar System.

In this paper, a new Interference Cancellation algorithm (IC-algorithm) and an improved FastICA algorithm with $K$-means cluster are proposed to extract the weak object signals from the Passive Radar System. Firstly, we introduce a framework of QPSK modulation and Interference Cancellation algorithm (IC-algorithm) theory to get rid of the strong interference signal. Then, the $K$-means cluster algorithm and the improved FastICA algorithm are proposed for the weak object signals separation. Finally, we verify the performance of our algorithms by simulations. The experimental results demonstrate the effectiveness of the proposed algorithm.

The rest of the paper is organized as follows. In Section 2, we introduce the Interference Cancellation algorithm (ICalgorithm). In Section 3, the improved FastICA algorithm with $K$-means cluster is introduced. In Section 4, we introduce and discuss the experimental results. Finally, the conclusion is drawn in Section 5. 


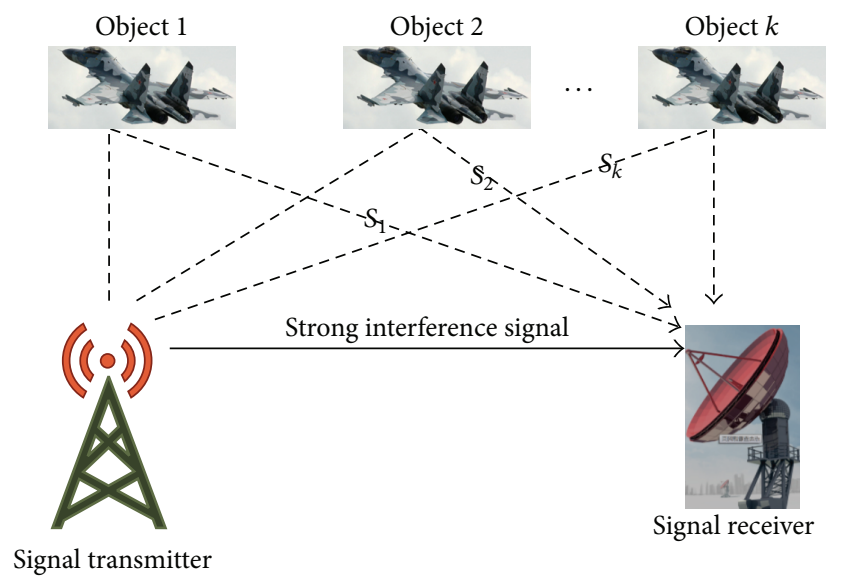

FIgURE 1: Passive Radar System obtains object signal information from the communication source.

\section{Interference Cancellation Algorithm (IC-Algorithm)}

In this section, we introduce the Interference Cancellation algorithm (IC-algorithm) to separate the weak object signals from the strong interference signal.

2.1. QPSK Modulation. QPSK (Quadrature Phase Shift Keying) is a type of phase shift keying, which contains two sinusoids (i.e., sine and cosine) that are used as the basic functions for the modulation [10]. The modulation is achieved by varying the phase of the basic functions and depends on the message symbols and can be formulated as [11]

$$
S_{\mathrm{QPSK}}(t)=A \cos \left(2 \pi f_{c} t+\theta_{n}\right), \quad n=1,2,3,4,
$$

where $A$ is the signal amplitude, $f_{c}$ is the signal frequency, and $\theta_{n}$ is the modulation phase. The constellation diagram of QPSK shows the constellation points lying on both $x$-axis and $y$-axis. This means that the QPSK modulated signal has an inphase component $(I)$ and also a quadrature component $(Q)$, since it has only two basic functions [12].

A QPSK modulator can be found in Figure 3. It is seen that there are two parts of QPSK, that is, QPSK modulated waveform in Figure 2 and QPSK modulated block in Figure 3 [10]. The implementation in Figure 3 is as follows: A demultiplexer is used to separate odd and even bits from the generated information bits. The signal on the in-phase arm is multiplied by cosine component and the signal on the quadrature arm is multiplied by sine component. QPSK modulated signal is obtained by adding the signal from both in-phase arm and quadrature arm [12].

2.2. Interference Cancellation Algorithm (IC-Algorithm). After the introduction of QPSK modulation, we introduce our Interference Cancellation algorithm (IC-algorithm).

In the Passive Radar System, the interference signal has very high power. Meanwhile, the mixed weak object signals are weak [13]. Here, we propose Interference Cancellation

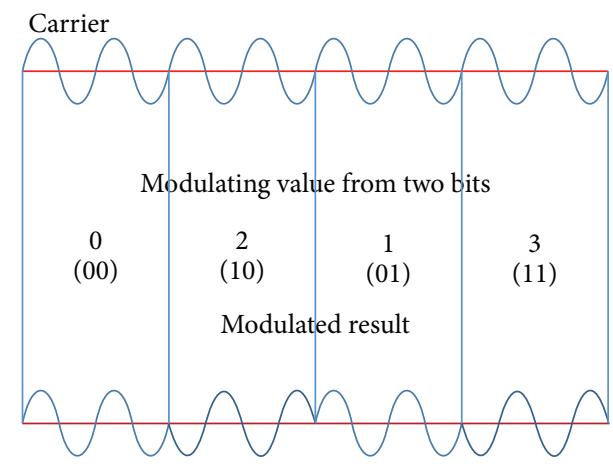

FIGURE 2: QPSK modulated waveform: the signal on the in-phase arm is multiplied by cosine component and the signal on the quadrature arm is multiplied by sine component.

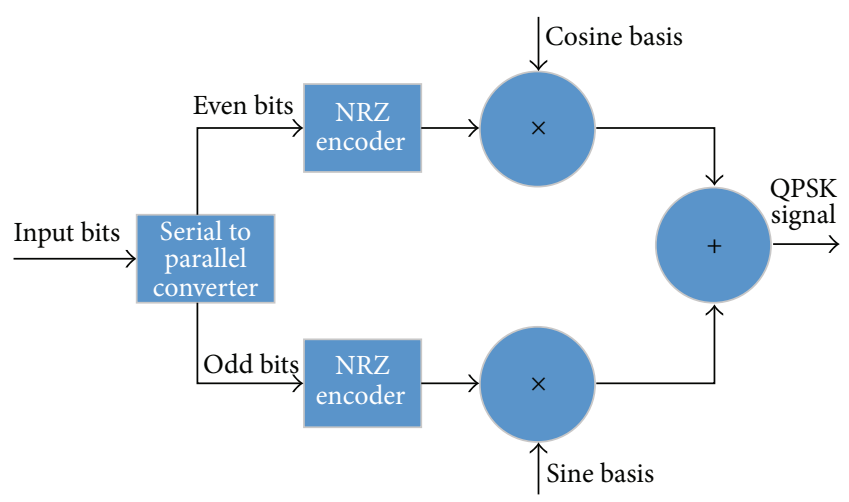

FIGURE 3: QPSK modulated block; QPSK modulated signal is obtained by adding the signal from both in-phase arm and quadrature arm.

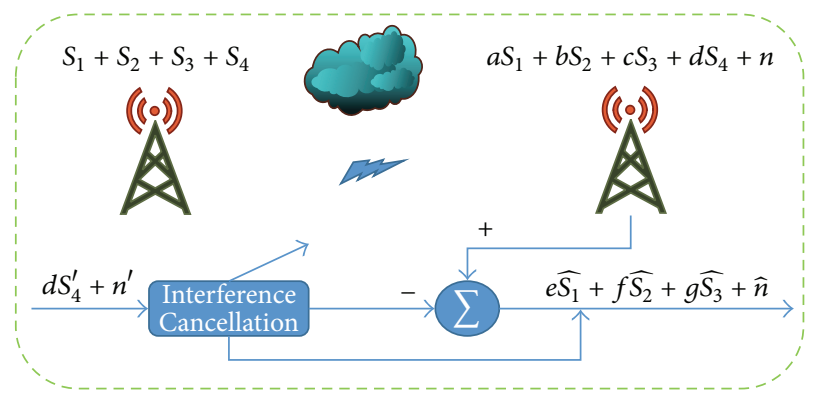

FIgURE 4: Framework of Interference Cancellation algorithm (ICalgorithm).

algorithm (IC-algorithm) to get rid of the jamming signal under the QPSK modulation and obtain the mixed weak object signals.

The framework of the Interference Cancellation algorithm (IC-algorithm) is displayed in Figure 4, where $S_{1}+$ $S_{2}+S_{3}+S_{4}$ is the original transmitted signal. $S_{1}, S_{2}$, and $S_{3}$ are the weak object signals. $S_{4}$ is the strong jamming. $a S_{1}+b S_{2}+c S_{3}+d S_{4}+n$ are the received mixed signals 
of $S_{1}+S_{2}+S_{3}+S_{4}$ through the Gauss channel [14]. Here, $n=\left[n_{1}, n_{2}, n_{3}, n_{4}\right]$ is the background noise, and

$$
\begin{aligned}
& a=\left[a_{1}, a_{2}, a_{3}, a_{4}\right], \\
& b=\left[b_{1}, b_{2}, b_{3}, b_{4}\right], \\
& c=\left[c_{1}, c_{2}, c_{3}, c_{4}\right], \\
& d=\left[d_{1}, d_{2}, d_{3}, d_{4}\right] .
\end{aligned}
$$

Assume there are four received signals, such as $l_{1}, l_{2}, l_{3}$, and $l_{4}$; then we have

$$
\left[\begin{array}{l}
l_{1} \\
l_{2} \\
l_{3} \\
l_{4}
\end{array}\right]=\left[\begin{array}{llll}
a_{1} & b_{1} & c_{1} & d_{1} \\
a_{2} & b_{2} & c_{2} & d_{2} \\
a_{3} & b_{3} & c_{3} & d_{3} \\
a_{4} & b_{4} & c_{4} & d_{4}
\end{array}\right] \cdot\left[\begin{array}{l}
S_{1} \\
S_{2} \\
S_{3} \\
S_{4}
\end{array}\right]+\left[\begin{array}{l}
n_{1} \\
n_{2} \\
n_{3} \\
n_{4}
\end{array}\right]
$$

$d S_{4}^{\prime}+n^{\prime}$ in Figure 4 is the reference strong jamming through the Gauss channel. Since $|d|\left\|S_{4}^{\prime}\right\| \gg\left\|n^{\prime}\right\|$, we obtain

$$
\frac{\left|d_{i}\right| \cdot\left\|S_{4}^{\prime}\right\|+\left\|n_{i}^{\prime}\right\|}{\left|d_{j}\right| \cdot\left\|S_{4}^{\prime}\right\|+\left\|n_{j}^{\prime}\right\|}=\frac{\left|\widehat{d}_{i}\right|}{\left|\widehat{d_{j}}\right|} \approx \frac{\left|d_{i}\right|}{\left|d_{j}\right|}, \quad i, j=1,2,3,4 .
$$

Because the reference strong jamming is known to us, we are able to estimate the channel parameters with the reference strong jamming. Then, the strong interference signal can be separated from the mixed signal based on the channel parameters. This process can be represented as

$$
\begin{aligned}
l_{1}- & \left(l_{1}+l_{2}+l_{3}+l_{4}\right) \cdot \frac{\widehat{d_{1}}}{\widehat{d_{1}}+\widehat{d_{2}}+\widehat{d_{3}}+\widehat{d_{4}}} \\
& \approx e_{1} S_{1}+f_{1} S_{2}+g_{1} S_{3}=\widehat{L_{1}}, \\
l_{2}- & \left(l_{1}+l_{2}+l_{3}+l_{4}\right) \cdot \frac{\widehat{d_{2}}}{\widehat{d_{1}}+\widehat{d_{2}}+\widehat{d_{3}}+\widehat{d}_{4}} \\
& \approx e_{2} S_{1}+f_{2} S_{2}+g_{2} S_{3}=\widehat{L_{2}}, \\
l_{3}- & \left(l_{1}+l_{2}+l_{3}+l_{4}\right) \cdot \frac{\widehat{d_{3}}}{\widehat{d_{1}}+\widehat{d_{2}}+\widehat{d_{3}}+\widehat{d_{4}}} \\
& \approx e_{3} S_{1}+f_{3} S_{2}+g_{3} S_{3}=\widehat{L_{3}},
\end{aligned}
$$

$$
\begin{gathered}
l_{4}-\left(l_{1}+l_{2}+l_{3}+l_{4}\right) \cdot \frac{\widehat{d_{4}}}{\widehat{d_{1}}+\widehat{d_{2}}+\widehat{d_{3}}+\widehat{d_{4}}} \\
\approx e_{4} S_{1}+f_{4} S_{2}+g_{4} S_{3}=\widehat{L_{4}} .
\end{gathered}
$$

Hence, we obtain the mixed useful signals $Y_{1}, Y_{2}$, and $Y_{3}$, and

$$
\left[\begin{array}{l}
Y_{1} \\
Y_{2} \\
Y_{3}
\end{array}\right]=\left[\begin{array}{lll}
e_{1} & f_{1} & g_{1} \\
e_{2} & f_{2} & g_{2} \\
e_{3} & f_{3} & g_{3}
\end{array}\right]\left[\begin{array}{l}
S_{1} \\
S_{2} \\
S_{3}
\end{array}\right]
$$

From the above process, the strong interference signal has been cancelled by (2)-(6). Finally, we obtain the mixed object signals denoted by $e \widehat{S_{1}}+f \widehat{S_{2}}+g \widehat{S_{3}}+\widehat{n}$ in Figure 4 . Furthermore, we can construct the vector space $\widehat{\mathscr{L}}=\left\{\widehat{L_{1}}, \widehat{L_{2}}\right.$, $\left.\widehat{L_{3}}, \widehat{L_{4}}\right\}$. Those vectors in this vector space satisfy the following properties [15].

(1) Closure of the addition:

$$
\begin{aligned}
& \forall x=\left(x_{1}, x_{2}, \ldots, x_{n}\right), y=\left(y_{1}, y_{2}, \ldots, y_{n}\right) \in \widehat{\mathscr{L}} \text {, and } \\
& \text { then } x+y=\left(x_{1}+y(1), x_{2}+y(2), \ldots, x_{n}+y(n)\right) \in \widehat{\mathscr{L}} .
\end{aligned}
$$

(2) Closure of the scalar multiplication:

$$
\begin{aligned}
& \forall x=\left(x_{1}, x_{2}, \ldots, x_{n}\right) \in \widehat{\mathscr{L}}, \forall \lambda \in R^{1}, \text { then, } \lambda x= \\
& \left(\lambda x_{1}, \lambda x_{2}, \ldots, \lambda x_{n}\right) \in \widehat{\mathscr{L}} .
\end{aligned}
$$

2.3. Discussions on the Properties of the Proposed Interference Cancellation Algorithm (IC-Algorithm). In this subsection, we state properties of proposed Interference Cancellation algorithm (IC-algorithm), such as computation complexity and convergence.

2.3.1. Complexity Analysis. The complexity of the introduced Interference Cancellation algorithm (IC-algorithm) can be specified by 2 parts [16].

(i) Estimate the channel parameters with the reference strong jamming.

In (3), suppose the coefficient matrix order is $L \times K$ and the source signal matrix order is $K \times N$; then the multiplication complexity is $O(K \times N \times L)$ and the addition complexity is $O(K \times N \times L)$.

(ii) Separate the strong interference signal from the mixed signal based on the channel parameters.

Equations (5) are also shown by matrix model as follows:

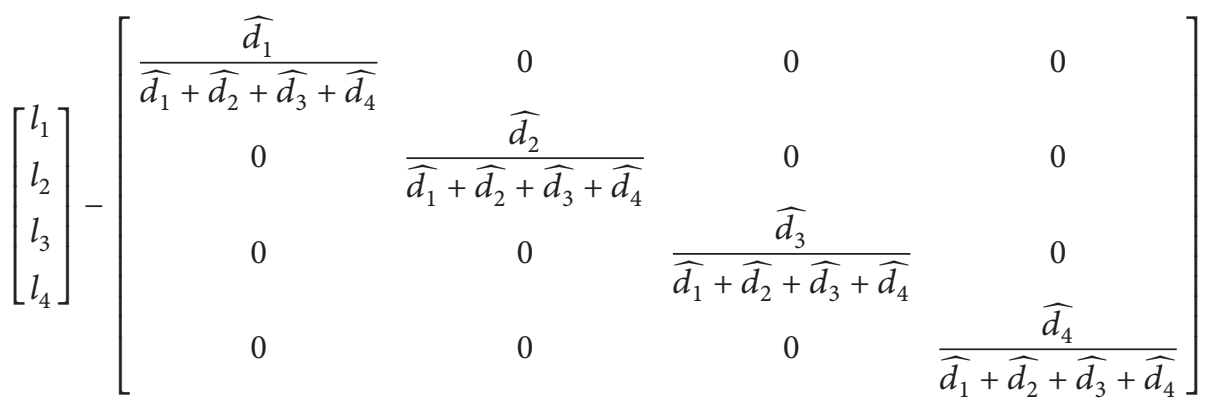




$$
\cdot\left[\begin{array}{l}
l_{1}+l_{2}+l_{3}+l_{4} \\
l_{1}+l_{2}+l_{3}+l_{4} \\
l_{1}+l_{2}+l_{3}+l_{4} \\
l_{1}+l_{2}+l_{3}+l_{4}
\end{array}\right]=\left[\begin{array}{ccc}
e_{1} & f_{1} & g_{1} \\
e_{2} & f_{2} & g_{2} \\
e_{3} & f_{3} & g_{3} \\
e_{4} & f_{4} & g_{4}
\end{array}\right] \cdot\left[\begin{array}{c}
S_{1} \\
S_{2} \\
S_{3} \\
S_{4}
\end{array}\right]
$$

In (7), suppose the received signal matrix order is $L \times N$, the coefficient matrix order is $L \times K$, and the received signal addition matrix order is $K \times N$; then, the multiplication complexity is $O(K \times N \times L)$ and addition complexity is $O(K \times$ $N \times L)$.

So, the overall complexity can be determined as $O(K \times$ $N \times L)$.

2.3.2. Convergence Analysis. In this subsection, we discuss the convergence of the proposed Interference Cancellation algorithm (IC-algorithm). Due to considering the influence of noise, the vector space $\widehat{\mathscr{L}}$ has an ambiguity. Obviously, the ambiguity will hamper the algorithm convergence, due to the arbitrary vectors influencing the iterative process [17]. A convergence point is assumed to be unstable under the Interference Cancellation algorithm (IC-algorithm) update rules if a small perturbation on the convergence procedure may cause the Interference Cancellation algorithm (IC-algorithm) to diverge away from the convergence point [18]. However, these can be easily avoided if each iteration minimizes $\left\|L_{i}-\widehat{L_{i}}\right\|$. The following statement discusses the convergence point of the Interference Cancellation algorithm (IC-algorithm).

Theorem 1. Let $\widehat{\mathscr{L}}=\left\{\widehat{L_{1}}, \widehat{L_{2}}, \widehat{L_{3}}, \widehat{L_{4}}\right\}$ denote the vector estimation space; then, for any initialization of the Interference Cancellation algorithm (IC-algorithm), the limit $\lim _{i \rightarrow \infty}$ exists; that is, the Interference Cancellation algorithm (IC-algorithm) is convergent [19].

Proof. Construct monotonic increasing sequence $\left(L_{1 n}, L_{2 n}\right.$, $\left.L_{3 n}, L_{4 n}\right) \in \widehat{\mathscr{L}}$. The sequence has an upper bound because noise has a little disturbance. Then, we have $\forall \varepsilon>0, \exists N$; when $n>N,\left|\left\|\left(L_{1 n}, L_{2 n}, L_{3 n}, L_{4 n}\right)\right\|-\left\|\left(L_{10}, L_{20}, L_{30}, L_{40}\right)\right\|\right|<\varepsilon$; that is,

$$
\lim _{n \rightarrow \infty}\left(L_{1 n}, L_{2 n}, L_{3 n}, L_{4 n}\right)=\left(L_{10}, L_{20}, L_{30}, L_{40}\right) .
$$

From the above procedure, we prove the convergence of the proposed Interference Cancellation algorithm (IC-algorithm).

\section{Improved FastICA Algorithm through $K$-Means Cluster}

In this section, we separate each interesting object signal with an improved FastICA algorithm combined with $K$-means clustering.

3.1. FastICA Algorithm. The FastICA algorithm is a popular procedure for blind source separation [20]. The size of the
Gaussian character is usually measured by negative entropy and can be written as [21]

$$
N_{g}(Y)=H\left(Y_{\text {Gauss }}\right)-H(Y),
$$

where $Y_{\text {Gauss }}$ is the random variable with the same covariance and $H(Y)$ is the formula for entropy calculation, which is defined as [22]

$$
H(Y)=-\int P_{Y}(\xi) \log P_{Y}(\xi) d \xi .
$$

Here, $P_{Y}(\xi)$ is the probability density function. The detailed process of the FastICA algorithm can be concluded as follows.

(1) Standardize data.

(2) Choose the original vector $W_{0}$ and set $\left\|W_{0}\right\|=1$.

(3) Select a nonquadratic function; for example,

$$
\begin{aligned}
& g_{1}(y)=\tanh \left(a_{1} y\right), \\
& g_{2}(y)=y \exp \left(-\frac{y^{2}}{2}\right), \\
& g_{3}(y)=y^{3} .
\end{aligned}
$$

(4) Let

$$
W_{p}=E\left\{Z g\left(W_{p}^{T}\right)\right\}-E\left\{g^{\prime}\left(W_{p}^{T}\right)\right\} W_{0} .
$$

(5) Let

$$
W_{p}=W_{p}-\sum\left(W_{p}^{T} W_{j}\right) W_{j}, \quad j=1,2, \ldots, p-1 .
$$

(6) Let

$$
W_{p}=\frac{W_{p}}{\left\|W_{p}\right\|} .
$$

(7) If $W_{p}$ is a convergence, go to (8). Otherwise, return to (4).

(8) Suppose $p$ is the number of the current extraction signals and $m$ is the number of the source numbers; let $p=p+1$; if $p \leq m$ return to (2).

Although FastICA algorithm is efficient, the performance heavily depends on the selection of the original vector $W_{0}$ $[23,24]$. Here, we improve the original FastICA algorithm by using the $K$-means for setting $W_{0}$, which is introduced in the next section. 

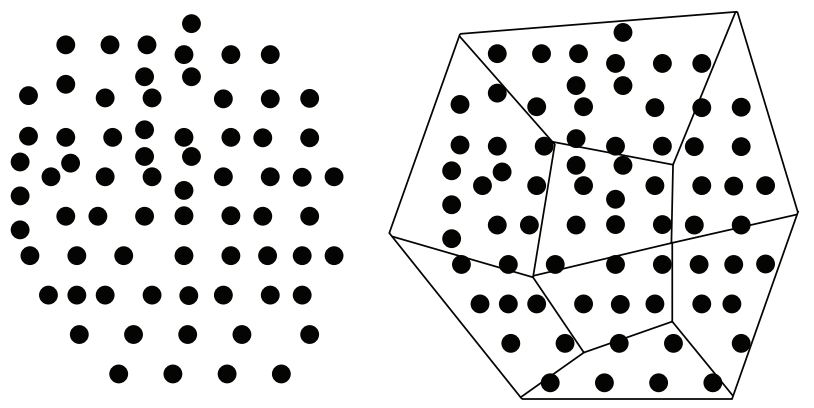

FIGURE 5: Primary data and the classification.

3.2. Improved FastICA Algorithm with K-Means Algorithm. There is a rich and diverse history in $K$-means algorithm as it was independently discovered in different scientific fields by Steinhaus (1957) [25], Lloyd (proposed in 1957, published in 1982) [26], Ball and Hall (1967) [27], and MacQueen (1967) [28], and it is the most popular and the simplest partitional algorithm [29, 30].

$K$-means algorithm aims to classify or group out objects based on attributes or features into number of groups. The group is done by minimizing the sum of squares of distances between every datum and corresponding cluster center. The main steps of $K$-means algorithm are as follows [31-33]:

(1) provide an initial number, $K$, of clusters;

(2) compute the squared Euclidean distance $d$ from each object to each cluster and assign each object to the closest cluster;

(3) minimize Within-Cluster Sum of Squares (WCSS) in (13) and update the cluster center for each cluster;

(4) recalculate the squared Euclidean distance $d$ based on the new memberships;

(5) repeat steps (3) and (4) until there is no possibility to move the objects to clusters.

Given a set of observations $\left(X_{1}, X_{2}, \ldots, X_{N}\right)$, where each observation is an $N$-dimensional vector, the $K$-means clustering method aims to separate the $N$ observations into $K$ sets $\left(S_{1}, S_{2}, \ldots, S_{N}\right)(K \leq N)$ with regard to minimizing the function as follows [34]:

$$
\mathrm{WCSS}=\min \sum_{i=1}^{k} \sum_{X_{j} \in S_{i}}\left\|X_{j}-\mu_{i}\right\|^{2}
$$

where $\mu_{i}$ is the mean vector of $S_{i}$ cluster $i=1,2, \ldots, K$.

The output of the $K$-means is the means vector $\mu_{1}, \mu_{2}$, $\ldots, \mu_{K}$. The examples are shown in Figures 5 and 6. It is seen that $\mu_{i}(i=1,2, \ldots, K)$ are the cluster centers and stand for the general feature of the corresponding class. So, we choose the original vector $W_{0}$ in $\mu_{1}, \mu_{2}, \ldots, \mu_{K}$. The flowchart of the proposed algorithm is shown in Figure 7.

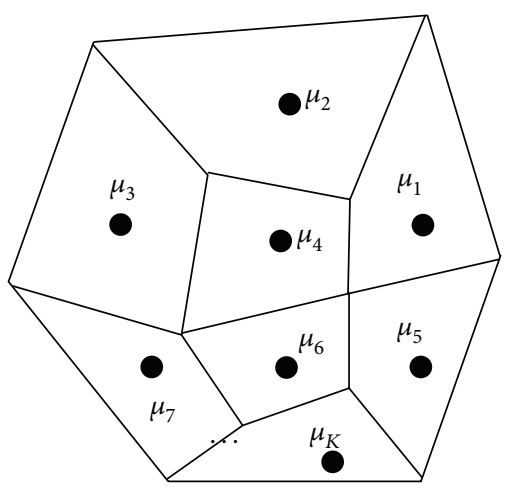

FIGURE 6: Cluster centers.

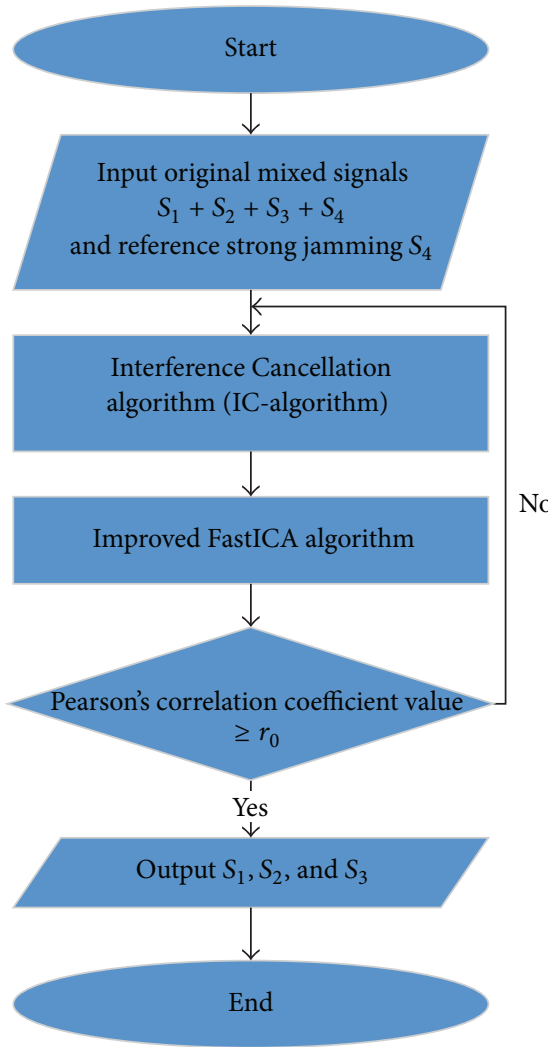

FIGURE 7: Flowchart of the proposed algorithm.

\section{Simulation and Blind Source Signal Separation Results}

In this section, we verify the proposed method. In the simulation, QPSK modulation signal will be separated from the mixed sensor signals.

We first introduce the parameter setting in our experiments. We set the sample rate as $f b=2 * 10^{4} \mathrm{~Hz}$, the transmission bit rate as $\mathrm{fb}=10^{3} \mathrm{bps}$, the modulation frequency as $f_{0}=2 * 10^{3} \mathrm{~Hz}$, the bit numbers as $m=80$, and the original signal numbers as $\mathrm{MK}=4$. 


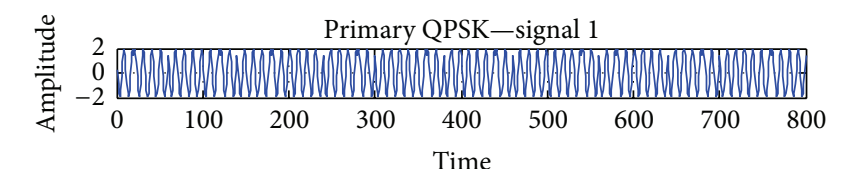

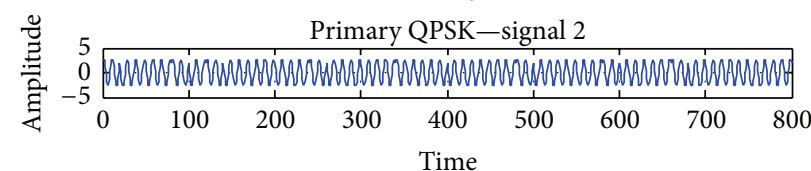

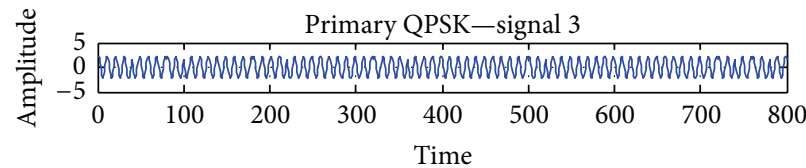

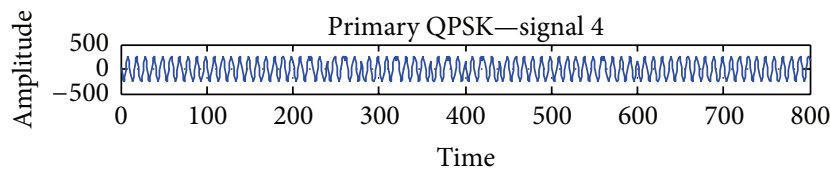

FIGURE 8: Source signals waves. Signal 1 to signal 3 are mixed weak object signal waveforms. Signal 4 is the strong interference signal waveform.
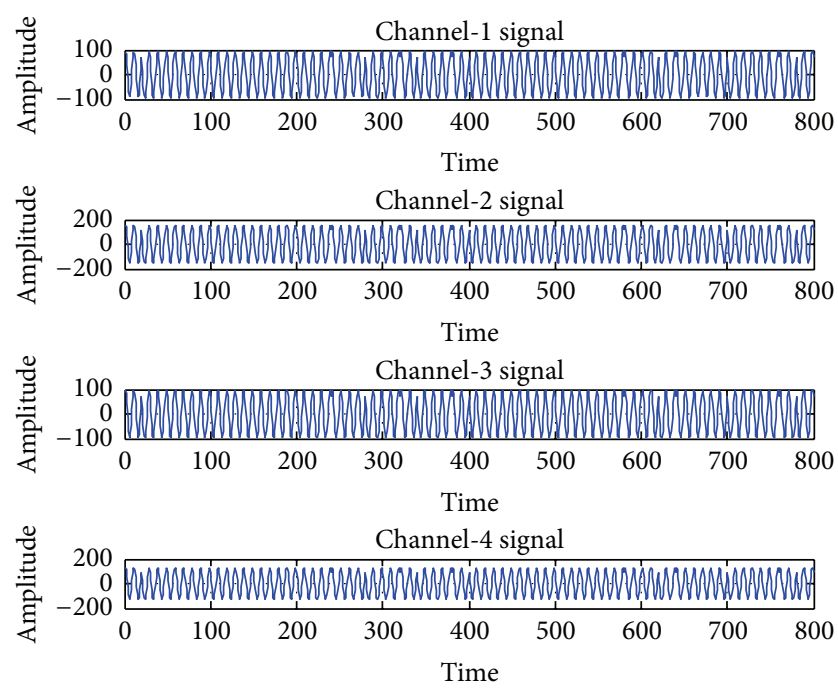

FIGURE 9: The received mixed signal waves after Gaussian channels. Four Gaussian channels are considered.

The sent original signal waveforms are shown in Figure 8. It is seen that signal 4 is the strong interference signal, while signal 1 to signal 3 are the weak object signals. We aim to separate each object signal from the sent source signals.

4.1. Channel Characteristic Estimation. After the Gauss channel based transitions, the received mixed signal waveforms are shown in Figure 9 (Received Composite Signal). Here, we consider four channels to fully simulate the realistic signal transmission, which are shown from top row to bottom row in Figure 9, respectively.

After using the proposed Interference Cancellation algorithm (IC-algorithm), the strong interference signal is

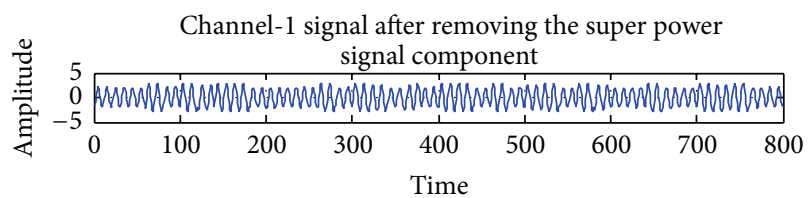

$$
\begin{array}{cccccccc|}
\text { Channel-2 signal after removing the super power } \\
\text { signal component }
\end{array}
$$$$
\begin{array}{ccccccccc}
\multicolumn{7}{c}{\text { Channel-3 signal after removing the super power }} \\
\text { signal component }
\end{array}
$$

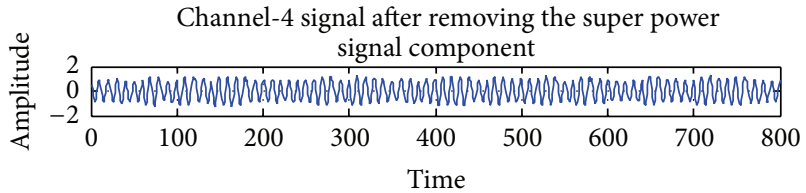

FIGURE 10: The four extracted weak object signal waveforms with the proposed Interference Cancellation algorithm (IC-algorithm).

removed from the four received mixed signal waves in Figure 8 . The corresponding four extracted mixed weak object signal waveforms are shown in Figure 10, respectively. In Figure 11, we further display the result of the strong interference signal channel parameter estimation error under different $S_{q} / N_{0}$; here $S_{q} / N_{0}$ is the ratio of the strong interference signal and background noise. The computational formula is composed of two steps.

(1) Vector standardization: suppose vector is $a=\left(a_{1}, a_{2}\right.$, $a_{3}$ ); the standardization vector is

$$
\frac{a}{\|a\|}=\left(\frac{a_{1}}{\|a\|}, \frac{a_{2}}{\|a\|}, \frac{a_{3}}{\|a\|}\right) .
$$

(2) Error function:

$$
\text { Error }=\left\|\frac{\widehat{a}}{\|\widehat{a}\|}-\frac{a}{\|a\|}\right\|_{2},
$$

where $\widehat{a}=\left(\widehat{a_{1}}, \widehat{a_{2}}, \widehat{a_{3}}\right)$ is the estimation of $a=\left(a_{1}, a_{2}\right.$, $\left.a_{3}\right)$.

It is seen that the Error becomes small along with the increase of $S_{q} / N_{0}$, which demonstrates the effectiveness of our Interference Cancellation algorithm (IC-algorithm).

The Interference Cancellation algorithm (IC-algorithm) includes four steps as follows.

(1) Set the factors.

(2) Generate the mixed signals.

(3) Mixed parameter estimation.

(4) Calculating error value.

The algorithm detail is provided in Appendix. 


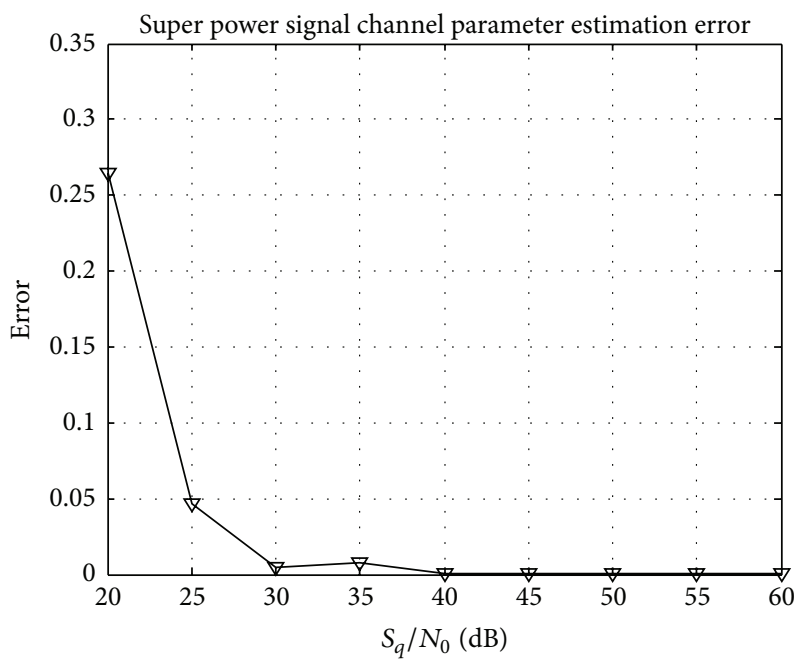

FIGURE 11: Influence of the strong interference signal on the mixed weak signals with the proposed Interference Cancellation algorithm (IC-algorithm).
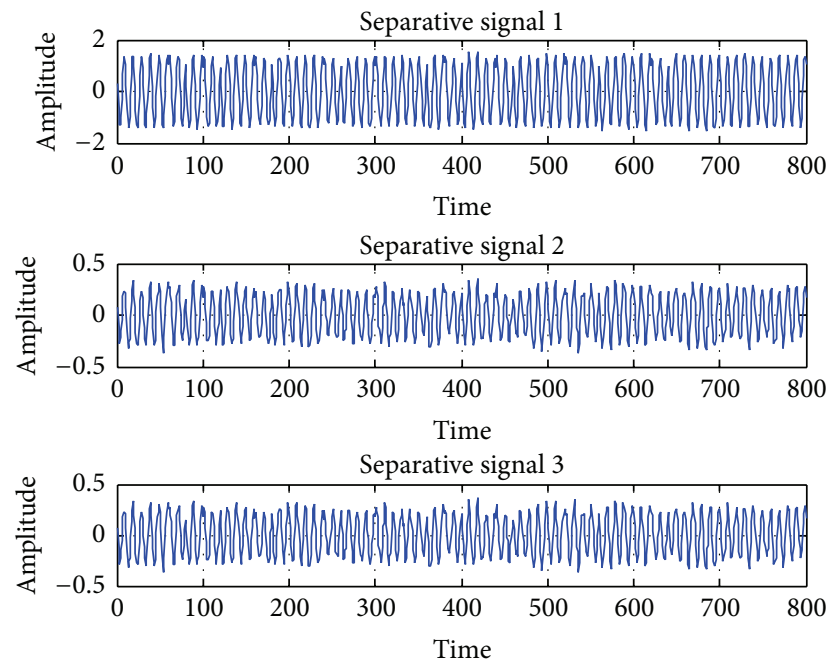

FIGURE 12: Blind source separation waveform using the proposed improved FastICA with $K$-means algorithm.

4.2. Simulations of the Separation Effect. The final blind source separation waveforms (the interested object signal) by the proposed improved FastICA with $K$-means algorithm are shown in Figure 12. The three signals are displayed. It is seen that the obtained three object signals are very similar to the initial object signals in Figure 8.

We compare the signals between Figures 12 and 8 by objective evaluation and further compare the separation performance with the classical FastICA algorithm [34]. Pearson's correlation coefficient value is used. The results are shown in

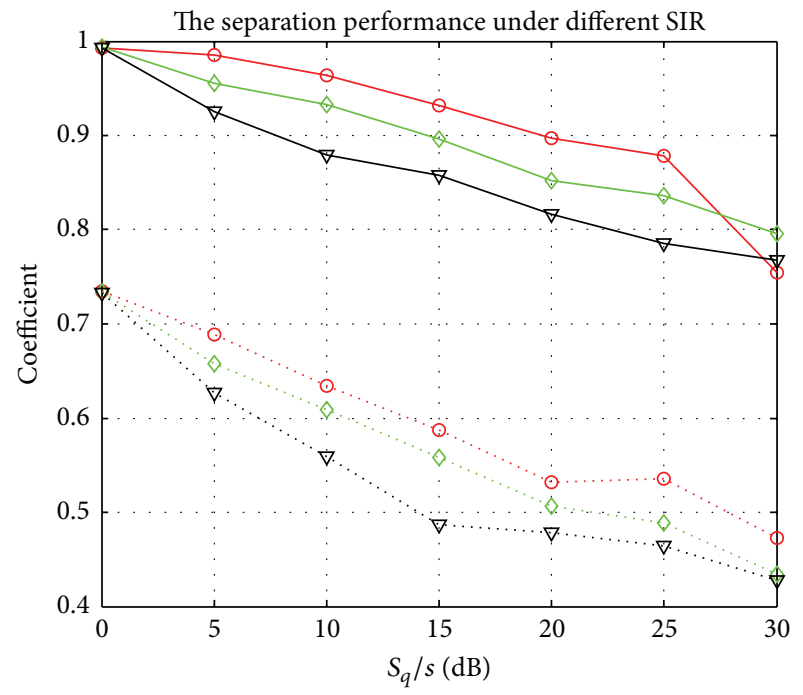

$$
\begin{aligned}
& - \text { Signal } 1 \text { (this paper's method) } \\
& \therefore \diamond . \text { Signal } 1 \text { (the classical FastICA method) } \\
& \diamond-\text { Signal } 2 \text { (this paper's method) } \\
& \therefore \quad \text { Signal } 2 \text { (the classical FastICA method) } \\
& \nabla-\text { Signal } 3 \text { (this paper's method) } \\
& \therefore \nabla \cdot \text { Signal } 3 \text { (the classical FastICA method) }
\end{aligned}
$$

FIGURE 13: Blind source separation result; this paper's method has a better performance than classical FastICA algorithm.

Figure 13, where Pearson's correlation coefficient is defined as follows [35]:

$$
r=\frac{\sum_{i=1}^{n}\left(x_{i}-\bar{x}\right)\left(y_{i}-\bar{y}\right)}{\sqrt{\sum_{i=1}^{n}\left(x_{i}-\bar{x}\right)^{2} \sum_{i=1}^{n}\left(y_{i}-\bar{y}\right)^{2}}} .
$$

We can see that blind sources signals can be efficiently separated by the proposed method, with a better performance than the classical FastICA algorithm.

\section{Conclusions}

In this paper, we suppose a special situation for blind source signal separation. Firstly, we propose an Interference Cancellation algorithm (IC-algorithm) to get rid of the jamming signal and the algorithm has a good performance. Then, we design an improved FastICA with $K$-means algorithm to improve the traditional FastICA algorithm, and the algorithm's robust performance can be markedly improved. The experiment results demonstrate the effectiveness of the proposed method.

\section{Appendix}

\section{The Detail of Interference Cancellation Algorithm (IC-Algorithm)}

See Algorithm 1. 
(1) Set the Factors

for $i=1: \mathrm{MK}$

if $i==$ list

$u=u u(p p)$

for $j j=1: 1000$ end

train_signal $(j j,:)=u * \operatorname{bpsk}\left(m, N, \operatorname{train}(j j,:), f_{0}, f_{s}\right)$;

$\operatorname{bpsk\_ signal}(i,:)=u * \operatorname{bpsk}\left(m, N, a(i,:), f_{0}, f_{s}\right)$;

else

$u 0=1$

$\operatorname{bpsk\_ signal}(i,:)=u 0 * \operatorname{bpsk}\left(m, N, a(i,:), f_{0}, f_{s}\right)$; end

end

$s=[]$

(2) Generate the Mixed Signals

for $i i=1: \mathrm{MK}$

if $i i \sim=$ list

$s 1=$ bpsk_signal $(i i,:)$;

$s=[s ; s 1]$

end

end

$A=\operatorname{rand}(\mathrm{MK}, \mathrm{MK})$;

$N_{0}=0.05 * \operatorname{randn}(\mathrm{MK}, N)$;

(3) Mixed Parameter Estimation

$B=\operatorname{zeros}(1, \mathrm{MK})$;

for $j j=1: 1000$

train $\_s=A(:$, list $) *$ train_signal $(j j,:)$;

$\operatorname{train} \_s=\operatorname{train} \_s+u_{-} n 0 * \operatorname{randn}(\mathrm{MK}, N)$;

$b 1 \_b 2=\operatorname{mean}\left(\operatorname{train} \_s(1,:) . / \operatorname{train} \_s(2,:)\right)$;

$b 1 \_b 3=$ mean $\left(\operatorname{train} \_s(1,:) . / \operatorname{train} \_s(3,:)\right)$;

$b 1 \_b 4=$ mean $\left(\operatorname{train} \_s(1,:) . / \operatorname{train} \_s(4,:)\right)$;

$b 2 \_b 3=\operatorname{mean}\left(\operatorname{train} \_s(2,:) . / \operatorname{train} \_s(3,:)\right)$;

$B B=\left[1 \quad 1 / b 1 \_b 21 / b 1 \_b 31 / b 1 \_b 4\right]$;

$B=B+B B$;

end

$B=B / 1000$;

(4) Calculating Error

if $\operatorname{norm}\left(B / \operatorname{norm}(B)-(A(\text {, , list }) / \operatorname{norm}(A(\text {, list })))^{\prime}\right)>0.01$

fprintf(');

elimate_error $(p p)=\operatorname{norm}\left(B / \operatorname{norm}(B)-(A(:, \operatorname{list}) / \operatorname{norm}(A(:, \text { list })))^{\prime}\right)$;

continue;

end

elimate_error $(p p)=\operatorname{norm}\left(B / \operatorname{norm}(B)-(A(:, \operatorname{list}) / \operatorname{norm}(A(:, \text { list })))^{\prime}\right)$;

Algorithm 1: Interference Cancellation algorithm (IC-algorithm) code.

\section{Competing Interests}

The authors declare that there is no conflict of interests.

\section{Authors' Contributions}

Lidong Zhu provided the instructions for system design for this research. Anhong Xie helped in performing the simulations. Zhongqiang Luo provided help in designing experiment scene. All the authors participated in the revisions of this paper.

\section{Acknowledgments}

This work is fully supported by a Grant from the National High Technology Research and Development Program of China (863 Program) (no. 2012AA01A502), the National
Natural Science Foundation of China (no. 61179006), and Science and Technology Support Program of Sichuan Province (no. 2014GZX0004).

\section{References}

[1] D. K. P. Tan, H. Sun, Y. Lu, M. Lesturgie, and H. L. Chan, "Passive radar using global system for mobile communication signal: theory, implementation and measurements," IEE Proceedings-Radar, Sonar and Navigation, vol. 152, no. 3, pp. 116-123, June 2005.

[2] L. Jian, L. Guoqing, and J. Nanzhi, "Airborn phased array radar: clutter and jamming suppression and moving target detection and feature rxtraction," in Proceedings of the IEEE Sensor Array and Multichannel Signal Processing Workshop, pp. 240-244, Cambridge, Mass, USA, March 2000. 
[3] J. Tsao and B. D. Steinberg, "Reduction of sidelobe and speckle artifacts in microwave imaging: the clean technique," IEEE Transactions on Antennas and Propagation, vol. 36, no. 4, pp. 543-556, 1988.

[4] P. T. Gough, "Fast spectral estimation algorithm based on the EFT," IEEE Transactions on Signal Processing, vol. 42, no. 6, pp. 1317-1322, 1994.

[5] I. Ziskind and M. Wax, "Maximum likelihood localization of multiple sources by alternating projection," IEEE Transactions on Acoustics, Speech, and Signal Processing, vol. 36, no. 10, pp. 1553-1560, 1988.

[6] H. Chen and H.-J. Su, "New approach to estimate DOA in presence of strong jamming/signal suppression," Acta Electronica Sinica, vol. 34, no. 3, pp. 530-534, 2006.

[7] C.-H. Yang, Y.-H. Shih, and H. Chiueh, "An $81.6 \mu \mathrm{W}$ FastICA processor for epileptic seizure detection," IEEE Transactions on Biomedical Circuits and Systems, vol. 9, no. 1, pp. 60-71, 2014.

[8] A. Dermoune and T. Wei, "FastICA algorithm: five criteria for the optimal choice of the nonlinearity function," IEEE Transactions on Signal Processing, vol. 61, no. 8, pp. 2078-2087, 2013.

[9] A. Hyvärinen, "Fast and robust fixed-point algorithms for independent component analysis," IEEE Transactions on Neural Networks, vol. 10, no. 3, pp. 626-634, 1999.

[10] S. Diao, Y. Zheng, Y. Gao et al., "A 50-Mb/s CMOS QPSK/OQPSK transmitter employing injection locking for direct modulation," IEEE Transactions on Microwave Theory and Techniques, vol. 60, no. 1, pp. 120-130, 2012.

[11] R. H. Morelos-Zaragoza and S. Lin, "QPSK block-modulation codes for unequal error protection," IEEE Transactions on Information Theory, vol. 41, no. 2, pp. 576-581, 1995.

[12] R. Porter, V. Tadic, and A. Achim, "Blind separation of sources with finite rate of innovation," in Proceedings of the 22nd European Signal Processing Conference (EUSIPCO '14), vol. 9, pp. 136-140, Lisbon, Portugal, September 2014.

[13] S. Choi, D. Crouse, P. Willett, and S. Zhou, "Multistatic target tracking for passive radar in a DAB/DVB network: initiation," IEEE Transactions on Aerospace and Electronic Systems, vol. 51, no. 3, pp. 2460-2469, 2015.

[14] F. Colone, D. W. O’Hagan, P. Lombardo, and C. J. Baker, “A multistage processing algorithm for disturbance removal and target detection in passive bistatic radar," IEEE Transactions on Aerospace and Electronic Systems, vol. 45, no. 2, pp. 698-722, 2009.

[15] O. Dordevic, E. Levi, and M. Jones, "A vector space decomposition based space vector PWM algorithm for a three-level sevenphase voltage source inverter," IEEE Transactions on Power Electronics, vol. 28, no. 2, pp. 637-649, 2013.

[16] M. Ravasi and M. Mattavelli, "High-abstraction level complexity analysis and memory architecture simulations of multimedia algorithms," IEEE Transactions on Circuits and Systems for Video Technology, vol. 15, no. 5, pp. 673-684, 2005.

[17] S. Yang, Z. Yi, M. Ye, and X. He, "Convergence analysis of graph regularized non-negative matrix factorization," IEEE Transactions on Knowledge and Data Engineering, vol. 26, no. 9, pp. 2151-2165, 2014.
[18] E. Oja and Z. Yuan, "The fastICA algorithm revisited: convergence analysis," IEEE Transactions on Neural Networks, vol. 17, no. 6, pp. 1370-1381, 2006.

[19] B. Jacob and M. R. Baiju, "A new space vector modulation scheme for multilevel inverters which directly vector quantize the reference space vector," IEEE Transactions on Industrial Electronics, vol. 62, no. 1, pp. 88-95, 2015.

[20] Z. Koldovský, P. Tichavský, and E. Oja, "Efficient variant of algorithm FastICA for independent component analysis attaining the Cramér-Rao lower bound," IEEE Transactions on Neural Networks, vol. 17, no. 5, pp. 1265-1277, 2006.

[21] L.-D. Van, D.-Y. Wu, and C.-S. Chen, "Energy-efficient FastICA implementation for biomedical signal separation," IEEE Transactions on Neural Networks, vol. 22, no. 11, pp. 1809-1822, 2011.

[22] A. Porta, S. Guzzetti, N. Montano et al., "Entropy, entropy rate, and pattern classification as tools to typify complexity in short heart period variability series," IEEE Transactions on Biomedical Engineering, vol. 48, no. 11, pp. 1282-1291, 2001.

[23] T. Wei, "A convergence and asymptotic analysis of the generalized symmetric FastICA algorithm," IEEE Transactions on Signal Processing, vol. 63, no. 24, pp. 6445-6458, 2015.

[24] M. Chen and P. Zhou, "A novel framework based on FastICA for high density surface EMG decomposition," IEEE Transactions on Neural Systems and Rehabilitation Engineering, vol. 24, no. 1, pp. 117-127, 2016.

[25] H. Steinhaus, "Sur la division des corps matériels en parties," Bulletin de l'Academie Polonaise des Sciences, vol. 4, no. 12, pp. 801-804, 1957 (French).

[26] S. P. Lloyd, "Least squares quantization in PCM," IEEE Transactions on Information Theory, vol. 28, no. 2, pp. 129-137, 1982.

[27] G. H. Ball and D. J. Hall, "A clustering technique for summarizing multivariate data," Behavioral Science, vol. 12, no. 2, pp. 153-155, 1967.

[28] J. MacQueen, "Some methods for classification and analysis of multivariate observations," in Proceedings of the 5th Berkeley Symposium on Mathematical Statistics and Probability, vol. 1, pp. 281-296, University of California Press, 1967.

[29] S. Lee, C. H. Park, and J. H. Chang, "Improved gaussian mixture regression based on pseudo feature generation using bootstrap in blood pressure estimation," IEEE Transactions on Industrial Informatics, 2015.

[30] J. Abawajy, M. Chowdhury, and A. Kelarev, "Hybrid consensus pruning of ensemble classifiers for big data malware detection," IEEE Transactions on Cloud Computing, 2015.

[31] M. Balouchestani, L. Sugavaneswaran, and S. Krishnan, "Advanced K-means clustering algorithm for large ECG data sets based on K-SVD approach," in Proceedings of the 9th International Symposium on Communication Systems, Networks and Digital Signal Processing (CSNDSP '14), pp. 177-182, Manchester, UK, July 2014.

[32] G. Logeswari, D. Sangeetha, and V. Vaidehi, "A cost effective clustering based anonymization approach for storing PHR's in cloud," in Proceedings of the 4th International Conference on Recent Trends in Information Technology (ICRTIT '14), pp. 1-5, Chennai, India, April 2014. 
[33] A. Sarkar and U. Maulik, "Rough based symmetrical clustering for gene expression profile analysis," IEEE Transactions on Nanobioscience, vol. 14, no. 4, pp. 360-367, 2015.

[34] J. Wan, S. Tu, C. Liao, S. Chen, and Z. Zhu, Theory and Technology on Blind Source Separation of Communication Signals, National Defense Industry Press, 2012.

[35] M. Pajovic and J. C. Preisig, "Performance analysis and optimal design of multichannel equalizer for underwater acoustic communications," IEEE Journal of Oceanic Engineering, vol. 40, no. 4, pp. 759-774, 2015. 


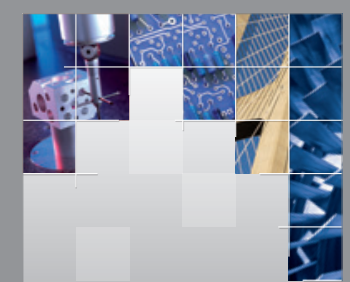

\section{Enfincering}
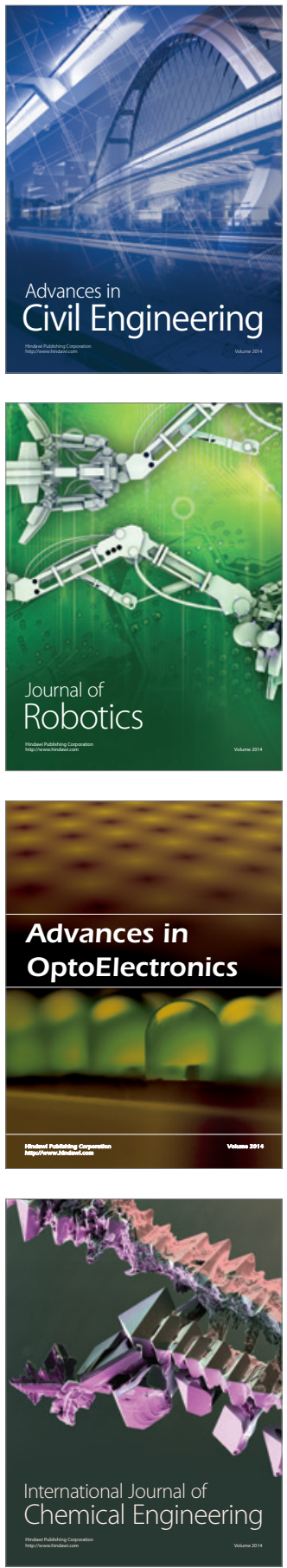

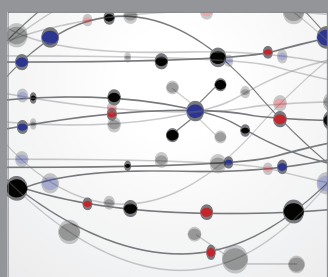

The Scientific World Journal

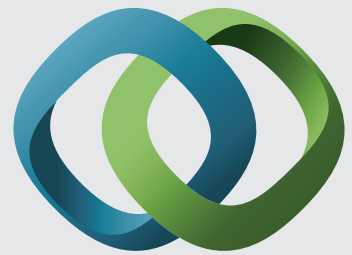

\section{Hindawi}

Submit your manuscripts at

http://www.hindawi.com
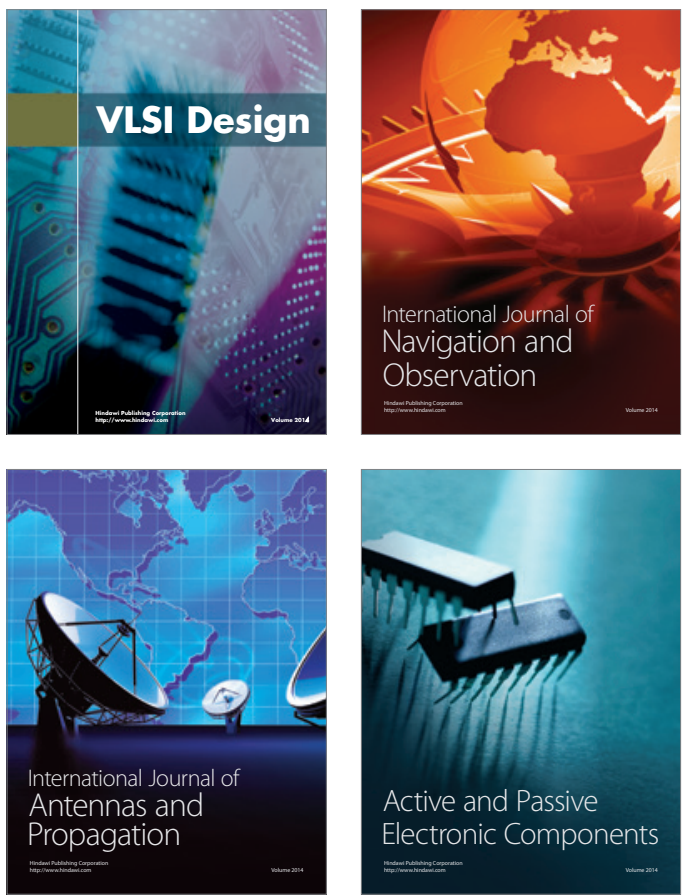
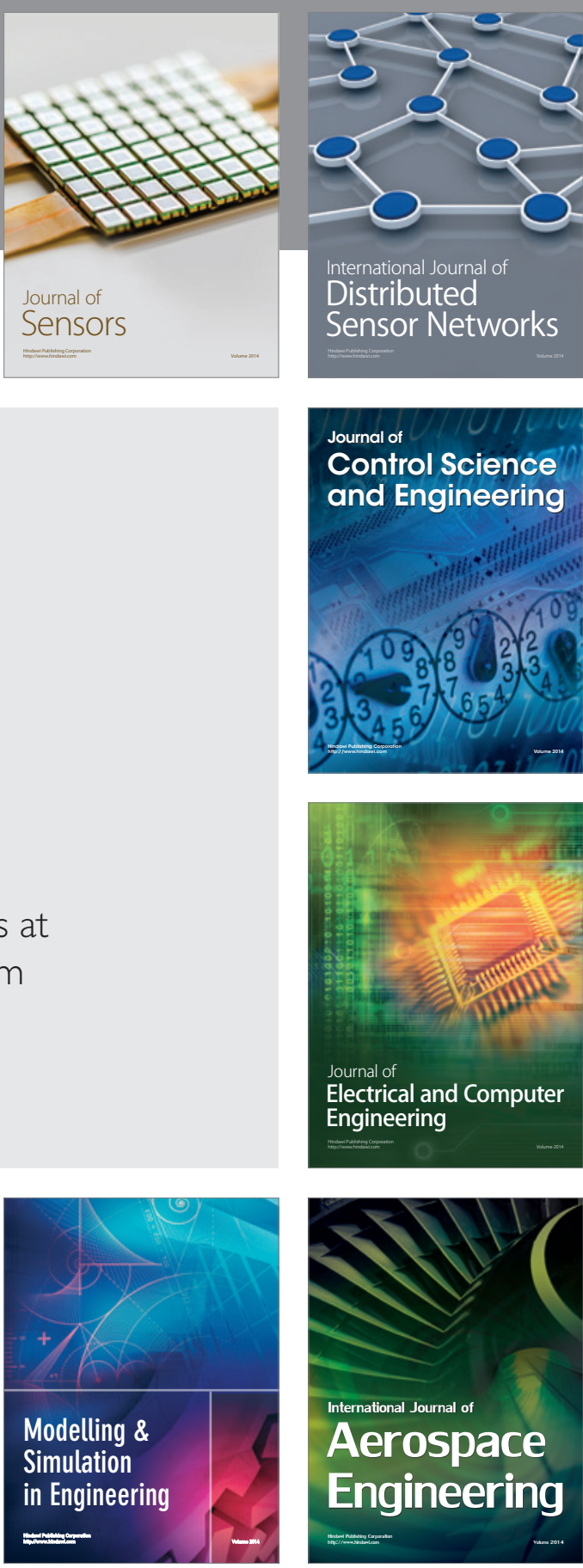

International Journal of

Distributed

Sensor Networks

Journal of

Control Science

and Engineering
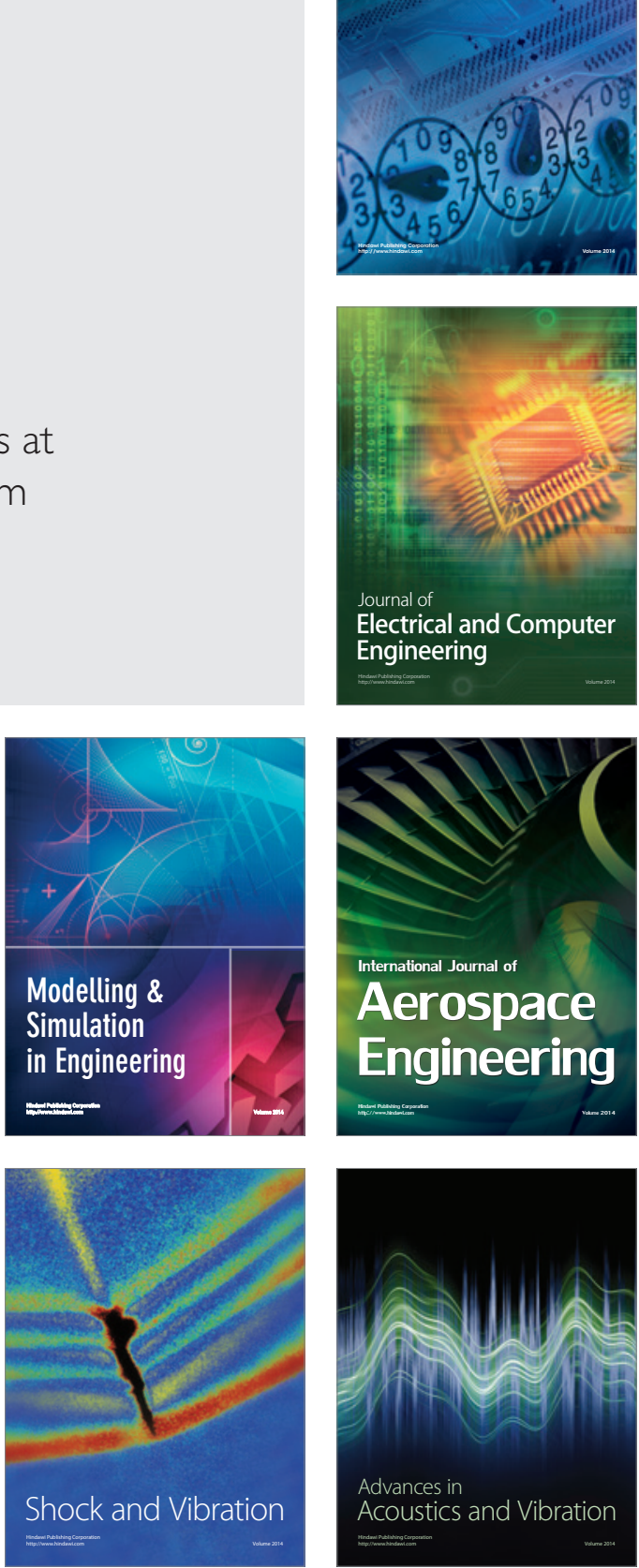